\title{
Thoracic and abdominopelvic actinomycosis
}

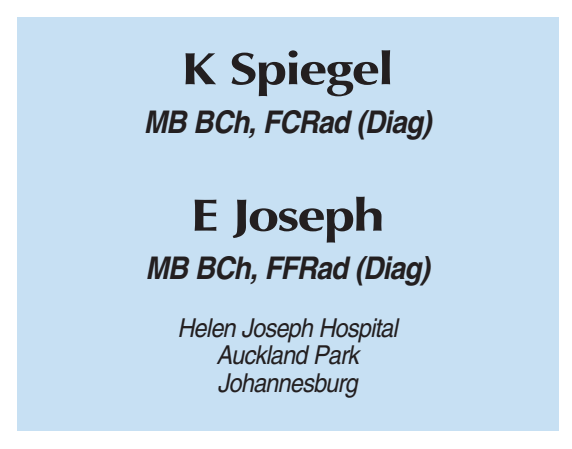

\begin{abstract}
Actinomycosis is a chronic infection caused by Gram-positive anaerobic bacteria which is found worldwide. Common features of actinomycosis infections include abscess formation, granulation, dense fibrous tissue formation, and the classical cutaneous sinuses with yellow/sulphur granular discharge. However some of the features may be nonspecific and the absence of classical features may delay the diagnosis. Since actinomycosis responds well to various antibiotic treatments, with or without surgical intervention it is worthwhile including actinomycosis infection in the differential diagnosis.

We present radiological findings for 4 patients presenting with different forms of thoracic and abdominopelvic actinomycosis infection, and a short review of the documented literature findings.
\end{abstract}

\section{Introduction}

Actinomycosis is a chronic infection caused by Gram-positive anaerobic bacteria, with the usual pathogen in man being Actinomycosis israelii, accounting for $85 \%$ of human infections. ${ }^{1,2}$ Actinomycosis is found worldwide and may affect any age group. ${ }^{1,3,4}$ This pathogen is normally found in the oral cavity and gastrointestinal tract (GIT) of healthy individuals. When the protective barriers are broken, opportunis- tic infection develops by direct extension, for example after dental manipulation. Infection may also develop by aspiration of oropharyngeal contents or gastrointestinal secretions causing respiratory tract infection, or may develop in association with foreign bodies such as intrauterine contraceptive devices (IUCDs) causing abdominopelvic acinomycosis. ${ }^{1,2,5}$ Haematogenous dissemination from a preexisting focus is rare, but may occur from thoracic disease. ${ }^{1,2,4}$ The chronic progressive suppurative infection caused by actinomycosis organisms has shown three major sites of predilection being cervicofacial (55\% mean frequency), ${ }^{3}$ abdominopelvic (25\%), and thoracic (15\%).

Common features of actinomycosis infections include abscess formation, granulation, dense fibrous tissue formation, and the classical cutaneous sinuses with 'yellow sulphur' granular discharge. Cavitations may be noted in lung involvement which may develop sinuses to the skin. Fistula formations are seen in GIT infections, and permeative bone destruction is seen with osseous involvement. However some of the features may be nonspecific and the absence of classical features may delay the diagnosis. ${ }^{1}$

Actinomycosis infection responds well to antibiotic treatment, traditionally intravenous Penicillin G for 4 - 6 weeks being the treatment of choice, followed by oral penicillin for 6 - 12 months. ${ }^{1}$ However erythromycin, tetracycline, cephalosporins and other antibiotics, alone or in combination, may also be used successfully. ${ }^{1,2,4,5}$ The duration of treatment may need to be tailored to the individual. A combined medical-surgical approach may be required. Surgical intervention assists in recovery but is not usually curative on its own. ${ }^{2}$

We present the radiological findings for 4 patients presenting with different forms of actinomycosis infection and give a short review of the relevant literature.

\section{Thoracic actinomy- cosis}

\section{Case 1}

A 63-year-old woman presented with chronic cough and chest pain. A small sinus was found on the skin adjacent to the sternum on the left which was not draining at the time of examination.

Chest X-ray demonstrated an illdefined mass adjacent to the aorta on the left, associated with left upper lobe fibrosis. An area of sub-segmental atelectasis was seen in the right lower lobe. This was thought not to be significant (Fig. 1).

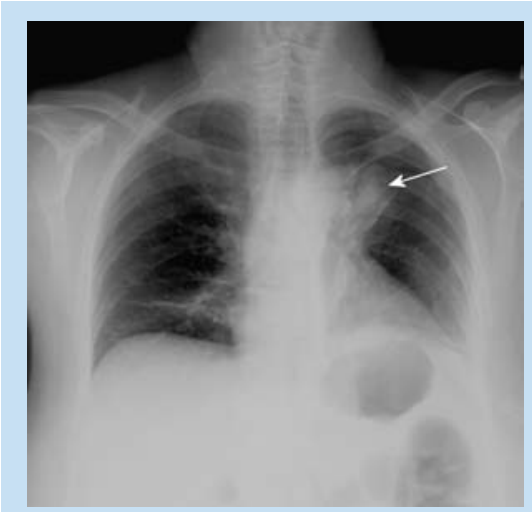

Fig. 1. Erect chest $X$-ray demonstrating opacification in the left upper lobe adjacent to the mediastinum (arrow).

A post-contrast CT scan of the chest demonstrated an irregular inhomogeneous soft-tissue mass in the left upper lobe adjacent to the aorta extending to the anterior thoracic wall lateral to the left edge of the sternum. The degree of contrast enhancement was negligible (Figs $2 \mathrm{a}$ and $\mathrm{b}$ ).

The differential diagnosis included tuberculosis, neoplasm, or actinomycosis.

An open lung biopsy was performed and the histology demonstrated sclerotic lung changes with interstitial fibrosis, and a filamentous bacterium, namely actinomycosis, was isolated.

\section{Case 2}

An adult male patient presented with swollen painful knees and a history of chronic cough. Chest X-ray demonstrated an irregular right upper lobe mass associat- 

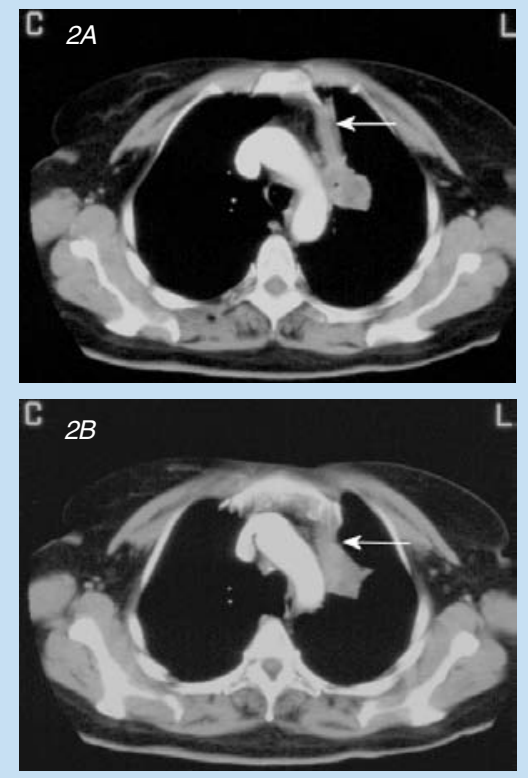

Figs 2a and b. Axial post-contrast CT scan of the chest demonstrating a left para-aortic mass extending to the anterior thoracic wall (arrow).
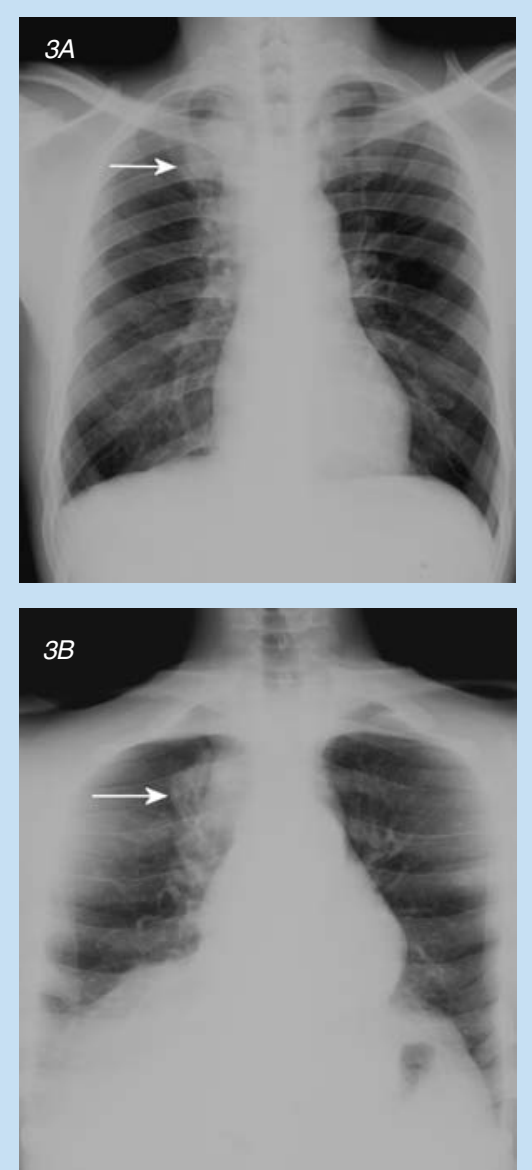

Figs $3 a$ and $b$. PA erect and lordotic (apical view) chest $X$-ray demonstrating the right upper lobe opacity in the right paratracheal region (arrow). ed with right upper-lobe volume loss and elevation of the right hilum as well as right parattracheal fullness (Figs $3 \mathrm{a}$ and $\mathrm{b}$ ). Contrast-enhanced CT scan of the chest confirmed the presence of the irregular right upper lobe posterior segment inhomogeneous lobulated mass with associated pleural reaction but no rib erosion. Extension into the mediastinum was demonstrated with a hypodense mass seen in the right paratracheal region which was splaying and displacing the major vessels. No significant contrast enhancement was demonstrated (Figs 4a and b).

Plain X-ray of the knees demonstrated
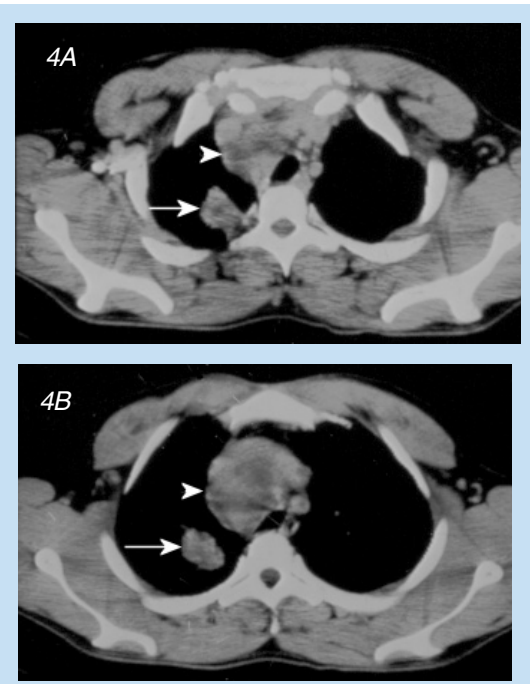

Figs $4 a$ and b. Axial non-enhanced CT scan of the chest demonstrating a lesion in the right upper lobe posterior segment (arrow) and an inhomogeneous hypodense lesion (arrow head) in the upper mediastinum.

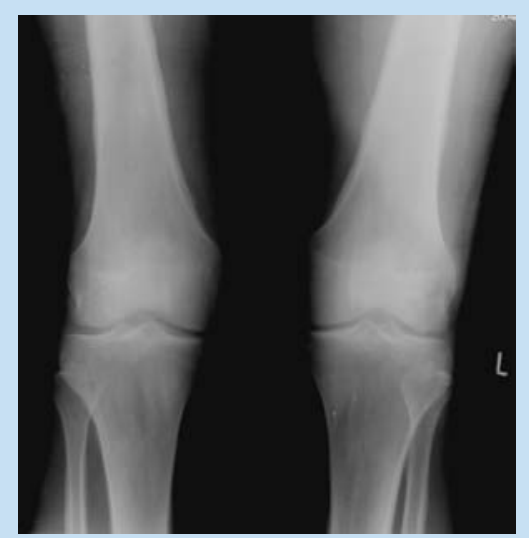

Fig. 5. AP X-ray of the knees demonstrating bilateral periostial reactions involving the femora, tibiae and fibulae. bilateral distal femoral and proximal tibial and fibular periosteal reactions bilaterally which were diagnosed as hypertrophic osteoarthropathy (Fig. 5).

The differential diagnosis in this case included right upper lobe neoplasm with mediastinal lymph node involvement, or a chronic infection with involvement of mediastinal lymph nodes, such as thoracic actinomycosis. Sputum analysis confirmed the diagnosis of actinomycosis.

\section{Abdominopelvic actinomycosis}

\section{Case 3}

A 42-year-old man presented with a palpable lesion in the right hypocondrium.

Chest X-ray demonstrated an elevation of the right hemi-diaphragm. There were no focal lung parenchymal lesions (Fig. 6).

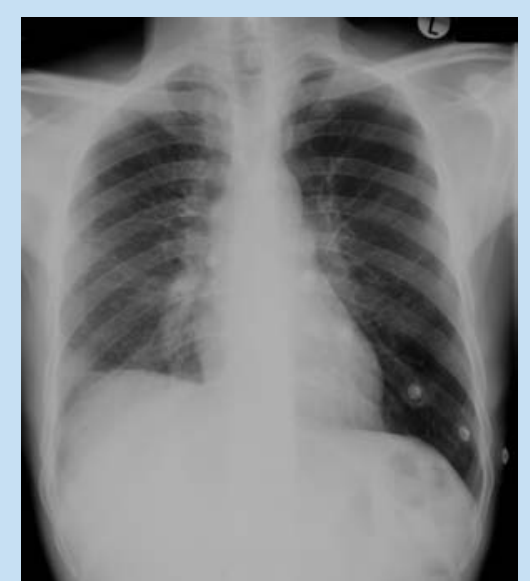

Fig. 6. Erect $P A$ chest $X$-ray demonstrating an apparent elevation of the right hemidiaphragm.

Contrast-enhanced CT scan of the chest demonstrated a subcutaneous abscess with communication to the pericapsular region of the liver extending through the anterior lower right chest wall. The lesion showed contrast rim enhancement. No focal pathology was demonstrated in the liver (Figs $7 \mathrm{a}$ and $\mathrm{b}$ ). This infection was pathologically proven to be actinomycosis and the patient duly responded to IV penicillin treatment. 

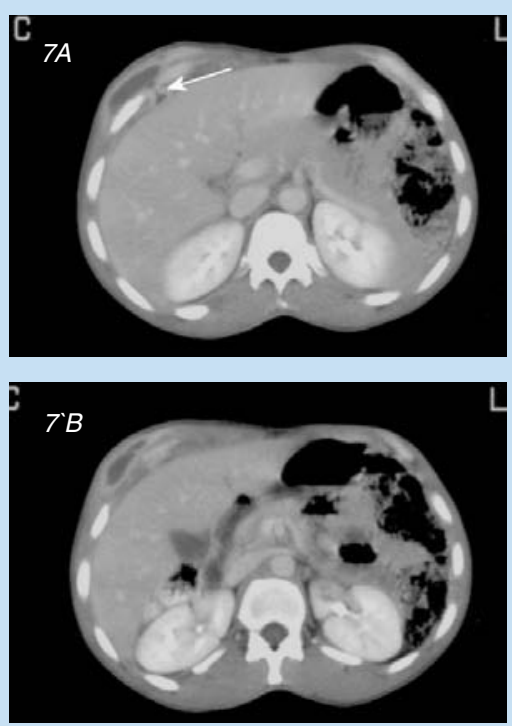

Figs $7 a$ and b. Axial post-contrast CT scan of the upper abdomen demonstrating a right upper-quadrant rim-enhancing collection over the right lobe of the liver anteriorly. Extension to the subcutaneous tissue in a collar stud appearance is demonstrated in Fig. 7a.(arrow).

\section{Case 4}

A 39-year-old woman presented with a pelvic mass. She had had an IUCD in situ for several years.

A contrast-enhanced CT scan of the abdomen demonstrated an inhomogeneous irregular pelvic mass involving the uterus with infiltration of the surrounding mesentery and multiple associated abscesses. Extension to the right anterior lower abdominal wall with abscess formation through the lower abdominal wall was shown, but with no breakthrough to the skin. The adjacent bowel wall was thickened (Figs 8a-d).

This was confirmed as actinomycosis and the patient responded well to treatment with penicillin.

\section{Discussion}

\section{Thoracic actinomycosis}

Thoracic actinomycosis is a rare disease that may mimic other pathology such as tuberculosis and primary or metastatic lung cancer. ${ }^{1,4}$ Actinomycosis may also coexist with these pathologies. ${ }^{2-4}$ Infection of the lung, pleura, mediastinum or chest wall may be caused by direct extension from cervicofacial infection, an
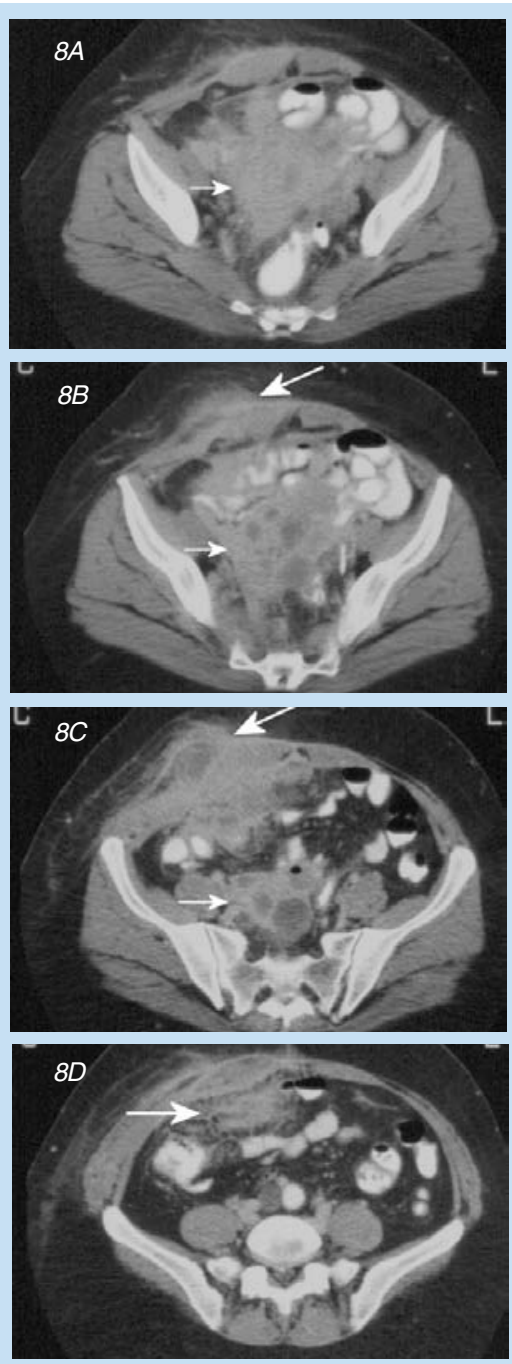

Figs 8a-d. Axial post-contrast CT scan of the pelvis demonstrating an inhomogeneously enhancing central pelvic mass iinvolving the uterus, with solid and liquid components representing multiple abscesses (small arrows) as well as infiltration of the surrounding mesentery and adjacent bowel wall thickening. Figs $c-d$ also demonstrate involvement of the right anterior lower abdominal wall with abscess formation (large arrows).

oesophageal tear, by inhalation or aspiration and rarely by haematogenous spread from a distant focus. ${ }^{2}$

The radiological features include localised ill-defined lung infiltrate, air space consolidation, or a mass lesion, with or without associated cavitation. ${ }^{2,4}$ A pulmonary infiltrate with a basal predominance and involvement of adjacent lobes through interlobar fissures is said to be suggestive of thoracic actinomycosis. ${ }^{1,4}$ The pathology may extend to the mediastinum, pericardium, myocardium, and preverte- bral space with vertebral body involvement. Extension to the skin surface with sinus tract formation may also be present, ${ }^{2}$ as demonstrated in case 1 (figs 1 and 2). Pleural involvement includes both pleural thickening and pleural effusion. ${ }^{1,6}$ The presence of a chronic pleural effusion with underlying lung changes and periosteal rib involvement is usually accepted as a diagnostic triad. Unfortunately Sumoza et al. ${ }^{4}$ found this to be the exception rather then the rule. Table I lists the common and less common features of thoracic actinomycosis. Not all features may be demonstrated by actinomycosis infection and in the absence of the classic cutaneous fistulas with yellow granular discharge the diagnosis may be delayed.

\section{Table I. Radiological features of thoracic actinomycosis}

1. Infiltrative changes suggestive of aspiration pneumonitis or consolidation extending across interlobar fissures.

2. Fibronodular or cavitary parenchymal disease .

3. An intraparenchymal mass.

4. Pleural effusion or empyema (rare).

5. Involvement of chest wall soft tissue and destruction of adjacent bone with formation of sinus tracts to the skin.

6. Involvement of the mediastinum, pericardium, myocardium. Rarely, superior vena caval obstruction and oesophageal fistula formation.

7. Thoracic vertebral destruction with preservation of disk space height.

8. Hypertrophic osteoarthropathy.

\section{Hypertrophic osteoarthropathy}

Hypertrophic osteoarthropathy (HOA) is characterised by periostosis of the tubular bones and digital clubbing. In the primary form no underlying cause is found, but when an underlying disease is present this syndrome is referred to as secondary 


\section{REVIEW ARTICLE}

HOA. Many underlying pathologies may be associated with HOA such as intrathoracic malignancy or infection, pleural pathology, cyanotic cardiac pathology, intestinal pathology, and more. The association of digital clubbing and HOA with chronic pulmonary disease was recognised in the late $1800 \mathrm{~s}^{7,8}$ In a review of the literature no reports of HOA with actinomycosis was found. Since thoracic actinomycosis is considered a chronic infection associated with cavitation, fibrosis and pleural involvement it falls under chronic pulmonary disease and therefore, as in our second case (case 2), it is assumed that it may be associated with HOA on this basis.

\section{Abdominopelvic actinomy- cosis}

Abdominal actinomycosis usually occurs following penetrating trauma, perforation of a hollow viscus (e.g. appendix) or surgical manipulation 9,10 There is a predilection for the ileocaecal region where it may be confused with caecal tuberculosis, amoeboma, chronic appendicitis, and carcinoma of the caecum. ${ }^{2,3}$ Primary pelvic actinomycosis may occur in association with IUCD colonisation and infection and is more likely with prolonged use of an IUCD, as demonstrated in case 4., ${ }^{3,11}$ Pelvic actinomycosis is also known to occur in association with septic abortion, retained sutures from previous surgery, or spread from an intra-abdominal focus.

Abdominopelvic actinomycosis may present as an aggressive-looking mass., ${ }^{3,5}$ Bowel-wall thickening with multiple abscesses, abundant granulation, dense fibrous tissue, as seen in case 4 (fig 8a-d), chronic fistulas and draining sinuses to the surface are the characteristic features. ${ }^{3,9,10}$ These features are not specific to actinomycosis and may mimic neoplasm, or other infective processes such as tuberculosis, or chronic appendicitis. ${ }^{2,10,12}$ The sinuses draining to the surface with yellow sulphur granules are also not unique to actinomy- cosis, and may occur in nocardiasis, chromomycosis and other fungal infections of the body. ${ }^{2}$ The aggressive infiltrative pattern of abdominopelvic actinomycosis has been described as an important radiological feature of this infection. ${ }^{3,5,13}$ Table II lists some of the imaging features of abdominopelvic actinomycosis.

\section{Table II. Radiological features of} abdominopelvic actinomycosis

1. GIT mucosal fold thickening and ulceration (may resemble Crohn's disease). ${ }^{3}$

2. Abdominal/pelvic infiltrative mass (often with multiple compartments involved).

3. Post-contrast CT may demonstrate homogeneous or inhomogeneous enhancement. ${ }^{3,5}$

4. Rupture of hollow viscus with free intra-abdominal air.

5. Chronic fistula and draining sinuses.

6. Retroperitoneal, psoas muscle or liver abscess formation.

7. Minimal or absent ascites. ${ }^{3}$

8. Stellate infiltrates from mass lesion with mesenteric involvement. ${ }^{5}$

\section{Conclusion}

In the absence of classical features of cutaneous sinuses with yellow sulphur granular discharge, the common imaging findings in abdominopelvic and thoracic actinomycosis may be nonspecific. Actinomycosis responds well to various antibiotic treatments, with or without surgical intervention. For this reason it is worthwhile including actinomycosis infection in the differential diagnosis, particu- larly where clinical findings do not support the diagnosis of other processes such as tuberculosis or neoplasm. Actinomycosis should also be considered in situations where there is prolonged IUCD use, previous pelvic or abdominal surgery or in appendicular infection.

\section{References}

1. Ossorio MA, Fields CL, Byrd RP, Roy TM Thoracic actinomycosis and human immunodeficiency virus infection. South Med J 1997; 90: 1136 1138 .

2. Smego RA jun., Foglia G. Actinomycosis. Clin Infect Dis 1998; 26: 1255-1263.

3. Lee I, Ha HK, Park CM, et al. Abdominopelvic actinomycosis involving the gastrointestinal tract: CT features. Radiology 2001; 220: 76-80.

4. Sumoza D, Raad I, Douglas E. Differentiating thoracic actinomycosis from lung cancer. Infect ions in Medicine 2000; 17: 695-698.

5. Ko SF, Ng SH, Lee TY, Lo CW. Retroperitoneal actinomycosis with intraperitoneal spread: stellate pattern on CT. Clin Imaging 1996; 20: 133-136.

6. Cheon JE, Lm JG, Kim MY, Lee JS, Choi GM, Yeon KM. Thoracic actinomycosis: CT findings (abstract). Radiology 1998; 209: 229-233.

7. Menard HA Hypertrophic osteoarthropathy eMedicine-hypertrophic osteoarthropathy http://www.emedicine.com/med/topic2929.htm April 2003.

8. Burton MD, Mark EJ. Case 46-1994- A 35 year-old smoker with an air fluid level in the upper lobe bulla. N Engl J Med 1994; 31: 1761-1767.

9. Goldwag S, Abbitt PL, Watts B. Case report: per cutaneous drainage of periappendiceal actinomycosis (abstract). Clin Radiol 1991; 44: 422-424.

10. Kaliaras V, Mylaria S, Lyra S, Tasonidou D, Thanos L. Ileo-caecal actinomycosis $2002 \mathrm{Dec} 27$ \{online\} URL: http://www.eurorad.org/ case. cfm?uid=2091luxembourg,euromultimedia

11. Yeguez JF, Mortinez SA, Sanda LP, Hellinger MD Pelvic actinomycosis presenting as malignant large bowel obstruction: a case report and a review of the literature (abstract). Am Surg 2000; 66(1): 8590.

12. Chaudhuri S, Billings PJ. Intra-abdominal actinomycosis presenting as complex abdominopelvic mass. J Indian Med Assoc 2002; 100: 463-464.

13. Ha HK, Lee HJ, Kim H, et al. Abdominal actinomycosis: CT findings in 10 patients (abstract). Am J Roentgenol 1993; 161: 791-794. 